\title{
Trastorno bipolar y trastorno por uso de sustancias. Estudio Madrid sobre prevalencia de patología dual
}

\section{Bipolar disorder and substance use disorders. Madrid study on the prevalence of dual disorders/pathology}

\author{
Francisco Arias*, Nestor Szerman**, Pablo Vega***, Beatriz Mesías***, Ignacio Basurte**, \\ DAVID RENTERO*. \\ *Servicio de Psiquiatría. Hospital Doce de Octubre. Madrid. **Servicio de Psiquiatría. Hospital Gregorio Marañon. Madrid. \\ ***Instituto de Adicciones. Madrid.
}

\section{Resumen}

La comorbilidad entre trastorno bipolar y trastorno por uso de sustancias (TUS) es de las más relevantes dentro del espectro de la patología dual por su prevalencia y sus repercusiones evolutivas y sociosanitarias. Nuestro objetivo fue estudiar las características de los pacientes con diagnóstico de trastorno bipolar y trastorno por uso de sustancias procedentes del Estudio Madrid sobre prevalencia de patología dual en sujetos en tratamiento en el servicio sanitario público. La muestra procede de pacientes en tratamiento en las redes públicas de salud mental y de tratamiento para las adicciones de la Comunidad de Madrid. Los sujetos fueron evaluados con la entrevista Mini International Neuropsychiatric Interview (MINI) para el diagnóstico de los trastornos mentales y con la escala Personality Disorder Questionnaire (PDQ) para el diagnóstico de los trastornos de personalidad. De los 837 pacientes incluidos, 174 tenían un diagnóstico a lo largo de la vida de trastorno bipolar, 83 con trastorno bipolar tipo I y 91 del tipo II. La mayoría de ellos tenían algún diagnóstico de TUS. En la red de salud mental, de los 208 participantes, 21 tenían un diagnóstico de trastorno bipolar, de estos el $13(61.9 \%)$ tenían un diagnóstico de TUS y se consideraron duales, mientras que en el resto de los pacientes de salud mental el $33.2 \%$ tenían un diagnóstico comórbido de TUS $(p=0.03)$. En los centros de drogas, de los 629 pacientes valorados, un $24.3 \%(n=153)$ tenían un diagnóstico de trastorno bipolar. El subgrupo de pacientes con trastorno bipolar tenía mayor prevalencia de adicción al alcohol y a la cocaína que el resto de los pacientes. Además, la edad de inicio en el consumo de alcohol era más precoz entre los adictos bipolares que entre los adictos no bipolares. Los bipolares duales era un subgrupo con mayor comorbilidad con trastornos de personalidad, otros trastornos mentales como trastornos de ansiedad y mayor riesgo de suicidio. Estos datos apoyan que el alcohol principalmente y la cocaína son las drogas más relacionadas con la presencia de un trastorno bipolar, pero al ser un estudio transversal no se pueden extraer conclusiones etiológicas. Palabras clave: Patología dual, prevalencia, trastorno bipolar, alcohol, cocaína, cannabis, opiáceos.

\section{Abstract}

Given its prevalence and impact on public health, the comorbidity of bipolar and substance use disorders is one of the most relevant of dual diagnoses. The objective was to evaluate the characteristics of patients from community mental health and substance abuse centres in Madrid. The sample consisted of 837 outpatients from mental health and substance abuse centres. We used the Mini International Neuropsychiatric Interview (MINI) and Personality Disorder Questionnaire (PDQ4+) to evaluate axis I and II disorders. Of these patients, 174 had a lifetime bipolar disorder, 83 had bipolar disorder type I and 91 had type II. Most patients had dual pathology. Of the 208 participants from the mental health centres, 21 had bipolar disorder and $13(61.9 \%)$ were considered dually-diagnosed patients, while $33.2 \%$ of non-bipolar patients had a dual diagnoses $(p=0.03)$. Of the 629 participants from the substance abuse centres, 153 patients (24.3\%) had a bipolar diagnosis. Bipolar dual patients had higher rates of alcohol and cocaine dependence than non-bipolar patients. Moreover, age at onset of alcohol use was earlier in bipolar duallydiagnosed patients than in other alcoholics. Bipolar dually-diagnosed patients had higher personality and anxiety disorder comorbidities and greater suicide risk. Thus, alcohol and cocaine are the drugs most associated with bipolar disorder. Given the nature of the study, the type of relationship between these disorders cannot be determined. Keywords: Dual diagnosis, prevalence, bipolar disorder, alcohol, cocaine, cannabis, opioids. 


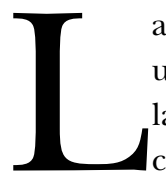

a patología dual se define como la presencia de un trastorno adictivo y otro trastorno mental a lo largo de la vida. Dicha concurrencia es habitual como señalan importantes estudios epidemiológicos en población general (Kessler, Nelson, McGonagle, Edlund, Frank y Leaf, 1996; Regier et al., 1990), e igualmente en muestras clínicas (Weaver et al., 2003). La comorbilidad modifica la presentación clínica y el curso evolutivo, repercute en el abordaje terapéutico al existir menos evidencia científica sobre el tratamiento más adecuado, además de relacionarse con una menor adherencia al tratamiento, un mayor uso de servicios sanitarios y mayor discapacidad funcional (González-Pinto et al., 2006; González-Pinto, Reed, Novick, Bertsch y Haro, 2010; Lagerberg et al., 2010).

En el estudio Epidemiologic Catchment Area (ECA) en población general, el trastorno mental del eje I más asociado con el consumo de drogas fue el trastorno bipolar (Regier et al., 1990), lo que ha sido confirmado en otros estudios en población general como el National Comorbidity Survey (NCS) o el National Epidemiologic Survey on Alcohol and Related Conditions (NESARC) y estudios clínicos posteriores (Kessler, Rubinow, Holmes, Abelson y Zhao, 1997; Grant et al., 2005; Merikangas et al., 2007; Cassidy, Ahearn y Carroll, 2001; Simon et al., 2004). Hay distintas explicaciones para justificar dicha alta prevalencia, como la hipótesis de la automedicación, factores de vulnerabilidad común o el consumo de sustancias como factor de riesgo (Levin y Hennessy, 2004). Se han descrito diferencias en los procesamientos de los refuerzos que pueden influir en la vulnerabilidad a la adicción (Singh et al., 2013). También se han considerado factores clínicos, los pacientes parecen consumir más en la fase de manía en posible relación con la búsqueda de placer de estos pacientes y las conductas desinhibidas e impulsivas propias de la manía, mientras que en las fases depresivas habría un menor consumo (Levin y Hennessy, 2004; Trost et al., 2014). La mayor impulsividad se considera característica de los trastornos bipolares (Powers et al., 2013). Por eso, es conveniente estudiar la prevalencia de las adicciones en este grupo a lo largo de la vida y actuales, dado que estas últimas pueden ser bastante variables dependiendo del estado clínico del paciente (McElroy et al., 2001). Posiblemente los modelos explicativos mencionados no son mutuamente excluyentes pues hay datos que apoyan a las distintas hipótesis y puede haber distintas explicaciones aplicable a un paciente en particular.

En general, en pacientes bipolares se ha referido un incremento en el consumo de todas las sustancias, pero hay drogas que pueden tener efectos diferenciales respecto a la regulación emocional y que puedan estar más relacionados con la inducción de cambios en el estado de animo de estos pacientes o que sean mas proclives a su consumo por un posible efecto beneficioso a corto plazo de sus alteraciones anímicas (Merikangas et al., 2008), por lo que no está claro si existen unas drogas de preferencia en los pacientes bipo- lares o son más prevalentes ciertas adicciones o si existen cambios en el patrón de consumo según el estado anímico predominante.

El objetivo de este estudio fue valorar la relación entre dependencia de drogas y el diagnóstico de trastorno bipolar en una muestra de pacientes ambulatorios en tratamiento en la red de salud mental y de drogas de la Comunidad de Madrid, dentro del estudio sobre prevalencia de patología dual en dicha Comunidad descrita en otros artículos (Arias et al., 2013a).

\section{Metodología}

\section{Muestra}

La pacientes fueron seleccionados consecutivamente por sus propios terapeutas en los centros de tratamiento de drogas (CAID y CAD: Centros de atención a las drogodependencias de la Comunidad de Madrid y del Ayuntamiento de Madrid respectivamente) (en adelante CD) y en los centros de salud mental (CSM) de la Comunidad de Madrid. Por lo tanto, se podían incluir tanto pacientes que acudían por primera vez como pacientes en revisión. Participaron 81 entrevistadores (psiquiatras, psicólogos o médicos generales con amplia experiencia en adicciones) de 64 centros de drogas de la Comunidad de Madrid y de 17 centros de salud mental. Todos los entrevistadores recibieron entrenamiento en la administración de la entrevista estructurada. Los participantes firmaron un consentimiento informado. El estudio fue aprobado por el Comité de Investigación Ética del Hospital Gregorio Marañon de Madrid. El porcentaje de participación fue del $87.2 \%$.

\section{Métodos}

Para establecer la presencia de trastornos mentales se utilizó la entrevista estructurada Mini Internacional Neuropsychiatric Interview (MINI) que permite diagnósticos según criterios DSM-IV y CIE-10, versión 5.0 (Sheehan et al., 1997). Es una entrevista que permite explorar los principales trastornos psiquiátricos del eje I actuales y a lo largo de la vida. Se ha comparado con entrevistas más largas como el SCID-I y el CIDI con puntuaciones de validez y fiabilidad aceptablemente elevados, pero requiere menos tiempo de aplicación y un breve entrenamiento para los entrevistadores clínicos (Sheehan et al., 1998; Amorim, Lecrubier, Weiller, Hergueta y Sheehan, 1998; Lecrubier et al., 1997). Para aquellos trastornos mentales que dicho instrumento no valora a lo largo de la vida, estos se exploraron a través de la entrevista clínica. Se estableció el diagnóstico de trastorno bipolar tipo I si en la entrevista MINI cumplía criterios de al menos un episodio maníaco actual o pasado, se estableció el diagnóstico de trastorno bipolar tipo II si cumplía criterios de al menos un episodio hipomaníaco y un episodio depresivo mayor actual o pasado pero no tenía criterios de episodio maníaco. 
Para establecer el diagnóstico de los trastornos de personalidad se utilizó la escala PDQ4+ (Personality Disorder Questionnaire) (Adaptación Española de Calvo, Caseras, Gutierrez y Torrubia, 2002). Este instrumento combina la rapidez y comodidad de uso de un cuestionario autoadministrado con el control del efecto de la sintomatología de estado de una entrevista. Es una buena herramienta diagnóstica siguiendo los criterios DSM-IV cuando se administra la escala de significación clínica.

\section{Análisis estadístico}

Se calcularon en todas la variables los parámetros descriptivos: media y desviación estándar en las cuantitativas que se ajusten a una distribución normal (test de Shapiro-Wilk) y en las que no se ajustan, la mediana y el rango intercuartílico. Las variables cualitativas se expresan mediante frecuencias relativas porcentuales. La comparación entre dos grupos se realizó mediante la prueba de chi-cuadrado ( $\chi 2)$ o la prueba exacta de Fischer si procedía para las variables categóricas y mediante la prueba t de Student o la U de Mann-Whitney para las variables cuantitativas. La variable principal se calcula en el intervalo de confianza para un $95 \%$ de seguridad. Los test se consideran significativos si la $\mathrm{p}<0,05$.

Para evaluar los factores que puedan influir en las predicción de la presencia de trastorno bipolar se aplica un modelo multivariante de regresión logística que incluye todos aquellos factores que pueden influir en la variable dependiente. Como criterios de selección del modelo se incluyen los parámetros que alcancen una $\mathrm{p}<0,1$ y como criterio de salida se establece $\mathrm{p}<0,05$; aunque se mantienen todos aquellos factores de confusión que modifiquen su $\beta$ o el de los otros parámetros en más de un $20 \%$. Además se testan las correspondientes interacciones, incluyéndose sólo las significativas. En caso de presencia de linealidad o colinealidad, se muestran los datos estratificados ajustados por las principales posibles variables de confusión. El análisis estadístico se genera mediante el programa SPSS v.17.

\section{Resultados}

Se incluyeron 837 pacientes: 208 (24.9\%) procedían de CSM y $629(75.1 \%)$ de CD. Del total de 837 pacientes evaluados, 710 tenían algún diagnóstico de trastorno por uso de sustancias (TUS) a lo largo de la vida (incluyendo el alcohol y excluyendo el tabaco). Había 127 sujetos (15.2\%) sin TUS. Del total de la muestra 174 pacientes tenían un diagnóstico de trastorno bipolar (TB) a lo largo de la vida (20.8\% del total de la muestra), 83 con diagnóstico de TB tipo I y 91 con TB tipo II.

\section{Características sociodemográficas}

En la tabla 1 se reflejan las características sociodemográficas de los pacientes bipolares. Eran más jóvenes que el resto
Tabla 1. Características de los pacientes con diagnóstico de trastorno bipolar a lo largo de la vida $(n=174)$

\begin{tabular}{ll}
\hline Edad (años) & $36.4(\mathrm{DE}=9.2)$ \\
\hline Varón & $126(72.8 \%)$ \\
\hline Soltero & $106(61.6 \%)$ \\
Casado & $38(22.1 \%)$ \\
Otros & $28(16.3 \%)$ \\
\hline Estudios primarios & $78(45.6 \%)$ \\
Secundarios & $69(40.4 \%)$ \\
Universitarios & $23(13.5 \%)$ \\
\hline Activo laboralmente & $80(46.2 \%)$ \\
Paro & $55(31.8 \%)$ \\
Otros & $38(21.0 \%)$ \\
\hline Convivencia con familia origen & $85(48.9 \%)$ \\
Convivencia con familia propia & $43(24.7 \%)$ \\
Solo & $29(16.7 \%)$ \\
Otros & $17(9.8 \%)$ \\
\hline Procedencia: Salud Mental $(\mathrm{n}=208)$ & $21(10.1 \%$ del total del CSM) \\
Centros de drogodependencias $(\mathrm{n}=629)$ & $153(24.3 \%$ del total de CD) \\
\hline Patología médica & $57(32.9 \%)$ \\
\hline Subtipos de T. bipolar: & $83(47.7 \%)$ \\
Tipo I & $91(52.3 \%)$ \\
\hline Tipo II &
\end{tabular}

Nota. $\mathrm{DE}=$ Desviación estándar

de la muestra (36.4 años, d.e. $=9.2$ frente a 38.9 , d.e. $=10.4$, $\mathrm{p}=0.004)$ y había predominio de varones, aunque como veremos esto difiere según procedan de CSM o CD.

\section{Prevalencia de trastornos por uso de sustancias (TUS) actual y a lo largo de la vida en la muestra de pacientes bipolares (Tabla 2).}

Dado que había predominio de pacientes procedentes de los CD, existe un alto porcentaje de TUS entre los pacientes bipolares. Había diferencias en la prevalencia de abuso o dependencia de alcohol, cocaína, cannabis y tabaco entre el grupo de bipolares y de no bipolares. Respecto a la dependencia de opiáceos no había diferencias. La edad de inicio en el consumo de alcohol fue menor en el grupo de pacientes bipolares que en los adictos no bipolares. Además había mayor prevalencia de antecedentes de consumo intravenoso de opiáceos entre estos pacientes bipolares.

\section{Diagnósticos de trastornos de personalidad (TP).}

Había predominio de diagnósticos de TP en el grupo de TB respecto al grupo de no bipolares $(n=113,64.9 \%$ vs $\mathrm{n}=301,45.4 \%$, respectivamente, $\mathrm{p}=0.001$ ), con diferencias significativas para todos los trastornos de personalidad valorados. Igualmente al agruparse por los distintos cluster, había predominio del cluster A, B y C en el grupo de TB.

\section{Modelo de regresión logística.}

Se realizó un modelo de regresión logística predictivo de la presencia de TB (Tabla 3). Al ajustarlo por edad, sexo y otras drogas, en el modelo sólo permanecieron como varia- 
Francisco Arias, Nestor Szerman, Pablo Vega, Beatriz Mesías, Ignacio Basurte, David Rentero

Tabla 2. Trastorno por uso de sustancias y trastornos de personalidad en la muestra de bipolares ( $n=174)$

\begin{tabular}{|c|c|c|}
\hline Droga & Bipolares & No bipolares \\
\hline Alcohol o TUS actual* & $143(82.2 \%)$ & $438(66.1 \%)$ \\
\hline A lo largo de la vida* & $165(94.8 \%)$ & $545(82.2 \%)$ \\
\hline A lo largo de la vida* & $134(77.0 \%)$ & $394(59.4 \%)$ \\
\hline TUS actual (sin alcohol)* & $121(69.5 \%)$ & $355(53.5 \%)$ \\
\hline A lo largo de la vida* & $145(83.3 \%)$ & $470(70.9 \%)$ \\
\hline Cocaína actual* & $94(54.0 \%)$ & $271(40.9 \%)$ \\
\hline A lo largo de la vida* & $121(69.5 \%)$ & $367(55.4 \%)$ \\
\hline Opiáceos actual+ & $18(10.3 \%)$ & $82(12.4 \%)$ \\
\hline A lo largo de la vida+ & $45(25.9 \%)$ & $167(25.2 \%)$ \\
\hline Cannabis actual** & $39(22.4 \%)$ & $94(14.2 \%)$ \\
\hline A lo largo de la vida** & $90(51.7 \%)$ & $263(39.7 \%)$ \\
\hline Sedantes actual+ & $8(4.6 \%)$ & $29(4.4 \%)$ \\
\hline Tabaco a lo largo de la vida* & $118(67.8 \%)$ & $372(56.1 \%)$ \\
\hline Diagnóstico de un solo TUS a lo largo de la vida & $34(19.5 \%)$ & $135(20.4 \%)$ \\
\hline Diagnóstico de mas de un TUS & $131(75.3 \%)$ & $410(61.8 \%)$ \\
\hline Uso intravenoso de opiáceos $(n=44)^{\star \star}$ & $35(79.5 \%)$ & $102(60.4 \%)$ \\
\hline \multicolumn{3}{|l|}{ Edad inicio consumo: } \\
\hline Alcohol ${ }^{* \star *}$ & $15.3(\mathrm{DE}=4.2)$ & $16.5(\mathrm{DE}=6.1)$ \\
\hline Cocaína+ & $20.3(\mathrm{DE}=6.2)$ & $21.2(\mathrm{DE}=6.5)$ \\
\hline 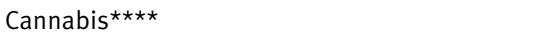 & $15.5(\mathrm{DE}=3.5)$ & $16.4(\mathrm{DE}=4.5)$ \\
\hline Opiáceos+ & $18.8(\mathrm{DE}=6.1)$ & $20.0(\mathrm{DE}=5.6)$ \\
\hline Trastornos de personalidad* & $113(64.9 \%)$ & $301(45.4 \%)$ \\
\hline
\end{tabular}

Nota. TUS = Trastorno por uso de sustancias. $\mathrm{DE}=$ desviación estándar.

$+=$ No significativo respecto a grupo de no bipolares. ${ }^{*}=p<0.001 .{ }^{* \star}=p<0.01 .{ }^{\star \star *}=p<0.05 .{ }^{* \star \star \star}=p<0.08$.

Tabla 3. Modelo de regresión logística predictivo de la presencia de trastorno bipolar.

\begin{tabular}{llll}
\hline Variable & Coeficiente $\beta$ & OR & P \\
\hline Edad & 0.02 & 0.98 & 0.05 \\
Sexo (Mujer) & 0.56 & 1.74 & 0.01 \\
Cocaína a lo largo de la vida & 0.50 & 1.65 & 0.01 \\
Alcohol a lo largo de la vida & 0.78 & 2.2 & 0.0001 \\
\hline
\end{tabular}

Nota. Chi-cuadrado del modelo=34.9, gl=4, $p=0.001$.

bles predictivas la adicción a cocaína como factor de riesgo $(0 \mathrm{R}=1,65, \mathrm{p}=0.01)$ y la adicción a alcohol como factor de riesgo $(\mathrm{OR}=2.2, \mathrm{p}=0.001)$. Dado que el modelo de regresión presentaba problemas de colinealidad por la relación entre las distintas adicciones se realizó un análisis estratificado por la presencia o no de la adicción a alcohol y cocaína. La relación entre alcohol y TB se mantuvo estadísticamente significativa tanto en presencia de la adicción a cocaína como en los no adictos a cocaína. Similares resultados se presentaron con la adicción a la cocaína al estratificarlo por la presencia de alcohol, aunque las diferencias no alcanzaron la significación estadística, pero sin diferencias entre los adictos al alcohol y los que no lo eran.

\section{Comparación entre bipolares tipo I y tipo II.}

No se objetivaron diferencias entre estos dos grupos. Había un leve mayor porcentaje de adicciones en el grupo de bipolar tipo I pero no estadísticamente significativos.

\section{Características de los pacientes bipolares adictos al alcohol.}

De los 174 pacientes bipolares, 92 tenían un diagnóstico de trastorno por uso de alcohol actual y 134 con trastorno a lo largo de la vida. Existía un predominio de varones $(79.1 \%)$ en este subgrupo respecto a los no adictos al alcohol $(65.9 \%, \mathrm{p}=0.05)$, mayor prevalencia de dependencia de cocaína y mayor frecuencia de trastornos de personalidad del cluster A. Sólo había 20 pacientes con dependencia de alcohol a lo largo de la vida sin otros TUS concomitantes, existiendo en este pequeño grupo un predominio de mujeres ( 11 mujeres, $55 \%$ y 9 varones, $45 \%$ ). Mientras que en el grupo de dependientes de alcohol con otras adicciones había predominio masculino (95 varones, $84.1 \%$ y 18 mujeres, $15.9 \%, \mathrm{p}=0.02$ frente a bipolares no adictos al alcohol).

\section{Características de los pacientes bipolares adictos a la cocaína.}

Había 94 pacientes con dependencia actual de cocaína y 121 con dependencia a lo largo de la vida. Había predomi- 
Trastorno bipolar y trastorno por uso de sustancias. Estudio Madrid sobre prevalencia de patología dual

Tabla 4. Trastornos bipolares $(n=21)$ en la red de salud mental $(n=208)$ y trastornos bipolares $(n=153)$ en la red de adicciones $(n=629)$

\begin{tabular}{|c|c|c|}
\hline & Red de Salud mental & Red de adicciones \\
\hline Edad & 42.7 años $(D E=13.3)+$ & 35.6 años $(D E=8.2)^{\star \star}$ \\
\hline Varones (total de bipolares) & $8(38.1 \%)+$ & $118(77.6 \%)^{\star}$ \\
\hline Mujeres & $13(61.9 \%)$ & $34(22.4 \%)$ \\
\hline Varones (bipolares duales) & $7(53.8 \%)$ & \\
\hline Mujeres & $6(46.2 \%)$ & \\
\hline Alcohol o TUS actual & $8(38.1 \%)+$ & \\
\hline A lo largo de la vida & $13(61.9 \%)+$ & \\
\hline Alcohol actual & $5(23.8 \%)+$ & $87(56.9 \%)^{\star \star}$ \\
\hline A lo largo de la vida & $10(47.6 \%)^{*}$ & $124(81.0 \%)^{\star \star}$ \\
\hline TUS actual (sin alcohol)+ & $5(23.8 \%)+$ & $116(75.8 \%)+$ \\
\hline A lo largo de la vida+ & $8(38.1 \%)+$ & \\
\hline Cocaína actual & $4(19.0 \%)^{\star}$ & $90(58.8 \%)+$ \\
\hline A lo largo de la vida & $4(19.0 \%)+$ & $117(76.5 \%)+$ \\
\hline A lo largo de la vida & 0 & $45(29.4 \%)+$ \\
\hline Cannabis actual & $3(14.3 \%)+$ & $36(23.5 \%)+$ \\
\hline A lo largo de la vida & $5(23.8 \%)+$ & $85(55.6 \%)+$ \\
\hline Sedantes actual & 0 & $8(5.2 \%)+$ \\
\hline A lo largo de la vida & $1(4.8 \%)$ & $31(20.3 \%)+$ \\
\hline Trastornos de personalidad* & $16(76.2 \%)^{\star}$ & $97(63.4 \%)^{\star \star \star}$ \\
\hline Trastornos de ansiedad & & $98(64.1 \%)^{\star \star \star}$ \\
\hline \multicolumn{3}{|l|}{ Subtipo de trastorno bipolar } \\
\hline Tipo I & 9 (4.3\% de pacientes de Salud Mental) & 74 (11.8\% de pacientes de adicciones) \\
\hline Tipo II & $11(5.3 \%)$ & $80(12.7 \%)$ \\
\hline \multicolumn{3}{|l|}{ Edad inicio consumo: } \\
\hline Alcohol & $16.4(\mathrm{DE}=3.2)+$ & $15.2(\mathrm{DE}=4.2)^{\star}$ \\
\hline Cannabis & & $15.3(\mathrm{DE}=3.3)^{\star \star \star \star}$ \\
\hline
\end{tabular}

Nota. TUS= Trastorno por uso de sustancias. $D E=$ desviación estándar.

$+=$ No significativo respecto a grupo de no bipolares. ${ }^{*}=p<0.05 .{ }^{* *}=p<0.01 .{ }^{* \star *}=p<0.001 .{ }^{* \star \star *}=p<0.07$.

nio masculino respecto a los otros bipolares $(\mathrm{p}=0.03)$, menor nivel educativo $(\mathrm{p}=0.05)$, mas situación de paro $(\mathrm{p}=0.05)$, mayor consumo de opiáceos y sedantes y mayor prevalencia de trastornos de personalidad del cluster B $(53.2 \%$ vs $37.5 \%$, $\mathrm{p}=0.04)$.

\section{Pacientes bipolares en la red de salud mental.}

De los 208 pacientes procedentes de la red de salud mental, había 21 con diagnóstico de TB. De estos, 13 (61.9\%) eran duales (frente a $62,33.2 \%$ en los no bipolares, $\mathrm{p}=0.03$ ). Había predominio femenino en el subgrupo de bipolares pero sin diferencias respecto al resto de pacientes del CSM, sin embargo al considerar los duales, había un ligero predominio masculino (de los 13 pacientes duales, 7 eran varones $(53.8 \%)$ y 6 mujeres). Al igual que en la muestra general, las drogas más asociadas con este diagnóstico fueron el alcohol y la cocaína y había mayor número de TP (Tabla 4).

\section{Pacientes bipolares en la red de drogas.}

De los 629 sujetos de la red de drogas, 153 eran bipolares $(24.3 \%)$. Aunque había predominio masculino en este subgrupo, el porcentaje de mujeres era superior que en el resto de la muestra del centro de drogas. La presencia de TB se asociaba con la dependencia de alcohol y con la mayor frecuencia de TP y diagnósticos de trastornos de ansiedad (Tabla 4).

Al comparar los pacientes bipolares de la red de drogas con la red de salud mental, había predominio de varones en la red de drogas $(\mathrm{p}=0.001)$, tenían peor situación laboral con más parados $(\mathrm{p}=0.001)$, más pacientes con patología médica comórbida ( $\mathrm{p}=0.05$ ) y más diagnósticos de trastorno por uso de alcohol, cocaína y cannabis. No había diferencias respecto a la prevalencia de TP comórbidos.

\section{Trastorno bipolar y riesgo de suicidio.}

Los pacientes con TB tenían mayor prevalencia de riesgo de suicidio valorado por la MINI que el resto de los pacientes $(p=0.001)$ y que el resto de pacientes duales $(p=0.006)$. Al estratificar por la presencia de trastorno por uso de alcohol, cocaína y cannabis a lo largo de la vida, la prevalencia del riesgo de suicidio era siempre superior en el grupo de bipolares frente a los no bipolares, pero si estaba presente uno de estos TUS la prevalencia de riesgo de suicidio era aún mayor. El riesgo de suicidio en estos pacientes bipolares se 
asoció con la presencia de mayor número de TP $(2.9$, de $=2.8$ vs 1.9 , de=2.7, $\mathrm{p}=0.01$ ), mayor número de diagnósticos del eje I (3.6, d.e. $=1.9$ vs 2.4 , d.e. $=1.5, \mathrm{p}=0.001)$, mayor número de diagnósticos de TUS (2.9, d.e.=1.6 vs 2.4 , d.e.=1.4) y una tendencia a una edad de inicio en el consumo de tabaco más precoz (13.6 años, d.e. $=3.1$ vs 15.1 , d.e. $=6.0, \mathrm{p}=0.08$ ) que los bipolares sin riesgo suicida.

\section{Discusión}

En la muestra de pacientes en tratamiento se observa una asociación del diagnóstico de TB con el TUS y fundamentalmente se observa una asociación con el alcohol y cocaína. Un alto porcentaje, hasta un $62 \%$, de los pacientes bipolares que acuden a tratamiento en la red de salud mental presentan un diagnóstico de TUS a lo largo de la vida. Cifras similares se han obtenido en otras muestras clínicas, como un $54 \%$ de prevalencia de TUS (Yatham, Kauer-Sant'Anna, Bond, Lam y Torres, 2009), un 59\% en una muestra de pacientes con manía (Frank, Boland, Novick, Bizzarri y Rucci, 2007) o un $72 \%$ de TUS a lo largo del a vida en bipolares ingresados (Bauer et al., 2005). Por otro lado, entre los pacientes adictos que acuden a centros de drogas para tratamiento, hasta una cuarta parte presenta un trastorno bipolar. Cifras similares a otras muestras clínicas (Nallet et al., 2013; Van Zaane, Van den Berg, Draisma, Nolen y Van den Brink, 2012).

El perfil sociodemográfico coincide con el de los pacientes duales, con predominio de varones y jóvenes (Tondo et al., 1999; Sonne y Brady, 1999). Sin embargo, en la red de salud mental ese predominio masculino es menos destacado y en la red de drogas existe de forma significativa un mayor número de mujeres con este tipo de patología dual que con el resto de diagnósticos, por lo que se puede considerar que las diferencias habituales por sexo en la patología dual, se atenúan en el caso de bipolares duales (Frye et al., 2003).

Las drogas más asociadas con el trastorno bipolar serían la dependencia de alcohol, cocaína y cannabis por orden de magnitud de la diferencia respecto a los no bipolares. Son drogas habitualmente descritas en la relación con el TB (Cerullo y Strakowski, 2007; Merikangas et al., 2008; Salloum et al., 2005). Además en el caso del alcohol, se observa que los pacientes con TB se inician en el consumo de alcohol a una edad más precoz lo que podría sugerir un papel causal para el consumo de alcohol. Aunque la naturaleza transversal del presente estudio no permite establecer otras relaciones etiológicas. Tanto el consumo de alcohol como de cocaína se han considerado sustancias que pueden inducir estados afectivos patológicos, pero también son sustancias que pueden ayudar a regular a corto plazo estados emocionales negativos aunque a largo plazo produzcan un efecto más perjudicial, e igualmente son sustancias cuyo consumo se incrementa durante las fases maníacas por el estado de exaltación y desinhibición presente en esos episodios. Así, la combinación de cocaína y alcohol parecen especialmente vinculadas con el trastorno bipolar por sus efectos específicos sobre el estado afectivo (Pacini et al., 2010) y se ha sugerido que se asocian con diferentes manifestaciones clínicas (Mitchell, Brown y Rush, 2007). Se ha sugerido que algunos pacientes bipolares con formas leves manifestarían la enfermedad de forma clínicamente más relevante con la exposición prolongada al alcohol (Cerullo y Strakowski, 2007). Otros autores observan relaciones entre el tipo de manifestaciones clínicas en la manía y el consumo específico de drogas como el alcohol o el cannabis (Güclü, Denormanci, Aydin, Erkiran y Köktürk, 2014) o el uso diferencial de drogas como la cocaína, alcohol o cannabis según el tipo de fase del trastorno bipolar (mixta, hipomanía, manía o depresión) (Maremmani et al., 2012). La elevada asociación entre trastorno bipolar y dependencia de alcohol se ha relacionado también con la posibilidad de factores genéticos comunes a ambos (Carmiol et al., 2015).

En el estudio realizado por nosotros en la submuestra de pacientes psicóticos, observamos una relación de la psicosis con la adicción al cannabis (Arias et al., 2013b), por lo que junto a estos datos sugiere que puede haber cierta especificidad entre distintas sustancias y distintos diagnósticos de trastornos mentales.

La alta prevalencia de diagnósticos de TP en estos pacientes duales concuerdan con otros estudios que señalan una elevada comorbilidad de estos en pacientes bipolares duales, pero también lleva a pensar si pueden existir problemas en el diagnóstico diferencial entre TP, manifestaciones propias de los TUS y del TB, como han señalado algunos autores (Levin y Hennessy, 2004; Stewart y El-Mallakh, 2007). Es posible que en esta muestra exista un sobrediagnóstico de TB por haber diagnosticado como tales a pacientes que presentan cambios del estado anímico propias de su TUS o $\mathrm{TP}$, e igualmente puede existir un sobrediagnóstico de TP en esta muestra por el instrumento utilizado, de cualquier forma el hecho de realizarse una entrevista estructurada puede minimizar ese sesgo e, igualmente, se ha referido que reconociendo la dificultad diagnóstica que conlleva estos cuadros y la necesidad de la realización de un adecuado diagnóstico diferencial entre las manifestaciones afectivas y conductuales de los TB, TP y TUS, la comorbilidad entre estos cuadros es habitual con otros TP u otros trastornos del eje I como los trastornos de ansiedad (Bauer et al., 2005; Merikangas et al., 2007; Mantere et al., 2006; Merikangas et al., 2011; Mazza et al., 2009). Así la comorbilidad del TB con TP como puede ser el trastorno límite se asocia con un mayor riesgo de TUS (Hidalgo-Mazzei, Walsh, Rosenstein y Zimmerman, 2015).

No está claro en la literatura que subtipo de TB está más relacionado con los TUS. Se ha referido que los pacientes con TB tipo I suelen tener más conductas adictivas (Merikangas et al., 2008) pero otros autores refieren que en los pacientes adictos son más habituales los cuadros hipomanía- 
cos o mixtos (Do y Mezuk, 2013; Himmelhoch, Mulla, Neil, Detre y Kupfer, 1976; Keller et al., 1986; Mazza et al., 2009). Nosotros no observamos diferencias respecto a la prevalencia de los distintos TUS, aunque la prevalencia de TUS era ligeramente mayor en los TB tipo I. Dado el pequeño número de pacientes bipolares procedentes de la red de salud mental no podemos extraer conclusiones sobre diferencias entre los subtipos de bipolares entre las dos redes asistenciales.

Nuestros datos sugieren que este subgrupo de bipolares adictos tiene mayor riesgo de suicidio que otros pacientes con trastornos mentales o adictivos en tratamiento, apoyando la mayor gravedad clínica de este subgrupo de pacientes duales. El incremento del riesgo de suicidio en los bipolares duales ya ha sido referido (Carrà, Bartoli, Crocamo, Brady y Clerici, 2014; Comtois, Russo, Roy-Byrne y Ries, 2004; González-Pinto et al., 2006). El subgrupo de pacientes con riesgo de suicidio además se asocian con mayor comorbilidad del eje I, de otros TUS y de mayor número de TP concomitantes, por lo que constituyen un subgrupo de pacientes que requiere especial vigilancia y manejo adecuado de todas estas comorbilidades.

Otro aspecto relevante del presente estudio es el distinto perfil de los pacientes bipolares duales entre las dos redes asistenciales públicas. En la red de drogas predominan pacientes bipolares con mayor comorbilidad adictiva y de otros trastornos del eje I, aunque de forma inesperada no del eje II, con mayor predominio masculino y peor situación laboral y médica. Los datos presentados en este trabajo serían extrapolables a la población bipolar de los centros de adicciones, aunque se requeriría un tamaño de muestra mayor para extrapolar estos datos a la red de salud mental.

Como limitaciones del estudio señalar que se trata de un estudio transversal por lo que no se pueden inferir relaciones causales. La mayoría de los pacientes son poliadictos por lo que no se pueden extraer conclusiones sólidas sobre el efecto de una droga en concreto. La muestra está compuesta por pacientes en tratamiento, por lo que no se puede extrapolar los datos a la población general y el hecho de padecer ciertos trastornos comórbidos pueden favorecer la búsqueda de tratamiento. La entrevista diagnóstica utilizada, la MINI, es posible que no sea la más adecuada en esta población, dado que no diferencia cuadros primarios de inducidos como realizan otras entrevistas diagnósticas o no realiza un diagnóstico diferencial adecuado, pero se escogió por la facilidad de la administración dado que estaban implicados un alto número de investigadores. La muestra procedente de la red de salud mental es muy pequeña. Dado que la mayoría de pacientes con trastorno bipolar acuden a esta última red, es posible que las características de nuestra muestra no sea representativa de los pacientes bipolares en tratamiento sino más bien de los adictos en tratamiento con comorbilidad con un trastorno bipolar.

Como fortalezas señalar que el tamaño muestral es destacado, sobre todo en lo referente a los procedentes de la red asistencial de drogas, por lo que consideramos que sería representativa de los pacientes adictos en tratamiento en las redes de adicciones. Además el uso de una entrevista estructurada incrementa la fiabilidad de los diagnósticos.

\section{Financiación}

Este trabajo fue financiado por la Obra Social Caja Madrid, el Instituto de Adicciones del Ayuntamiento de Madrid, la Agencia Antidroga de la Comunidad de Madrid y la Oficina Regional de Salud Mental de la Comunidad de Madrid. El proyecto fue promovido por la Sociedad Española de Patología Dual.

\section{Agradecimientos}

A todos los investigadores del Instituto de Adicciones de Madrid, de la Agencia Antidroga de Madrid y del Servicio Regional de Salud Mental que participaron en el proyecto.

\section{Conflicto de intereses}

Los autores declaran que no existe conflicto de intereses.

\section{Referencias}

Amorim, P., Lecrubier, Y., Weiller, E., Hergueta, T. y Sheehan D. (1998). DSM-IV-R Psychotic Disorders: procedural validity of the Mini International Neuropsychiatric Interview (MINI). Concordance and causes for discordance with the CIDI. European Psychiatry, 13, 26-34. doi: 10.1016/S0924-9338(97)86748-X

Arias, F., Szerman, N., Vega, P., Mesias, B., Basurte, I., Morant, C., ... Babín, F. (2013a). Estudio Madrid sobre prevalencia y características de los pacientes con patología dual en tratamiento en las redes de salud mental y de atención al drogodependiente. Adicciones, 25, 118 127.

Arias, F., Szerman, N., Vega, P., Mesias, B., Basurte, I., Morant, C., ... Babin, F. (2013b). Abuso o dependencia al cannabis y otros trastornos psiquiátricos. Estudio Madrid sobre prevalencia de patología dual. Actas Españolas de Psiquiatría, 41, 123-130.

Bauer, M.S., Altshuler, L., Evans, D.R., Beresford, T., Williford, W.O. y Hauger, R. (2005). Prevalence and distinct correlates of anxiety, substance, and combined comorbidity in a multi-site public sector sample with bipolar disorder. Journal of Affective Disorders, 85, 301-315.

Calvo, N., Caseras, X., Gutierrez, F. y Torrubia, R. (2002). Spanish version of the personality diagnostic questionnaire-4+ (PDQ-4+). Actas Españolas de Psiquiatría, 30, 7-13.

Carmiol, N., Peralta, J.M., Almasy, L., Contreras, J., Pacheco, A., Escamilla, M.A., ... Glahn, D.C. (2014). Shared genetic factors influence risk for bipolar disorder and 
alcohol use disorders. European Psychiatry, 29, 282-287. doi: 10.1016/j.eurpsy.2013.10.001

Carrà, G., Bartoli, F., Crocamo, C., Brady, K.T. y Clerici, M. (2014). Attempted suicide in people with co-occurring bipolar and substance use disorders: systematic review and meta-analysis. Journal of Affective Disorders, 167, 125135. doi: 10.1016/j.jad.2014.05.066

Cassidy, F., Ahearn, E.P. y Carroll, B.J. (2001). Substance abuse in bipolar disorder. Bipolar Disorders, 3, 181-188.

Cerullo, M.A. y Strakowski, S.M. (2007). The prevalence and significance of substance use disorders in bipolar type I and II disorder. Substance Abuse Treatment, Prevention, and Policy, 2, 29.

Comtois, K.A., Russo, J.E., Roy-Byrne, P. y Ries, R.K. (2004). Clinicians' assessments of bipolar disorder and substance abuse as predictors of suicidal behavior in acutely hospitalized psychiatric inpatients. Biological Psychiatry, 56, 757-763.

Do, E.K. y Mezuk, B. (2013). Comorbidity between hypomania and substance use disorders. Journal of Affective Disorders, 150, 974-980. doi: 10.1016/j.jad.2013.05.023

Frank, E., Boland, E., Novick, D.M., Bizzarri, J.V. y Rucci, P. (2007). Association between illicit drug and alcohol use and first manic episode. Pharmacology, Biochemistry and Behavior, 86, 395-400.

Frye, M.A., Altshuler, L.L., McElroy, S.L., Suppes, T., Keck, P.E., Denicoff, K., ... Post, R.M. (2003). Gender differences in prevalence, risk, and clinical correlates of alcoholism comorbidity in bipolar disorder. American Journal of Psychiatry, 160, 883-889.

González-Pinto, A., Mosquera, F., Alonso, M., López, P., Ramírez, F., Vieta, E. y Baldessarini, R.J. (2006). Suicidal risk in bipolar I disorder patients and adherence to longterm lithium treatment. Bipolar Disorders, 8, 618-624.

González-Pinto, A., Reed, C., Novick, D., Bertsch, J. y Haro, J.M. (2010). Assessment of Medication Adherence in a cohort of patients with bipolar disorder. Pharmacopsychiatry, 43, 263- 270. doi: 10.1055/s-0030-1263169

Grant, B.F., Stinson, F.S., Hasin, D.S., Dawson, D.A., Chou, S.P., Ruan, W.J. y Huang, B. (2005). Prevalence, correlates, and comorbidity of bipolar I disorder and axis I and II disorders: results from the National Epidemiologic Survey on Alcohol and Related Conditions. Journal of Clinical Psychiatry, 66, 1205-1215.

Güclü, O., Şenormancı, Ö., Aydın, E., Erkıran, M. y Köktürk, F. (2014). Phenomenological subtypes of mania and their relationships with substance use disorders. Journal of Affective Disorders, 174, 569-573. doi: 10.1016/j. jad.2014.11.016

Hidalgo-Mazzei, D., Walsh, E., Rosenstein, L. y Zimmerman, M. (2015). Comorbid Bipolar Disorder and Borderline Personality Disorder and Substance Use Disorder. Journal of Nervous and Mental Disease, 203, 54-57. doi: 10.1097/NMD.0000000000000235
Himmelhoch, J.M., Mulla, D., Neil, J.F., Detre, T.P. y Kupfer, D.J. (1976). Incidence and significance of mixed affective states in a bipolar population. Archives of General Psychiatry, 33,1062-1066.

Keller, M.B., Lavori, P.W., Coryell, W., Andreasen, N.C., Endicott, J., Clayton, P.J., ... Hirschfeld, R.M. (1986). Differential outcome of pure manic, mixed/cycling, and pure depressive episodes in patients with bipolar illness. The Journal of the American Medical Association, 255, 31383142.

Kessler, R.C., Nelson, C.B., McGonagle, K.A., Edlund, M.J., Frank, R.G. y Leaf, P.J. (1996). The epidemiology of cooccurring addictive and mental disorders: Implications for prevention and service utilization. American Journal of Orthopsychiatry, 66, 17-31.

Kessler, R.C., Rubinow, D.R., Holmes, C., Abelson, J.M. y Zhao, S. (1997). The epidemiology of DSM-III-R bipolar I disorder in a general population survey. Psychological Medicine, 27, 1079-1089.

Lagerberg, T.V., Larsson, S., Sundet, K., Hansen, C.B., Hellvin, T., Andreassen, O.A. y Melle, I. (2010). Treatment delay and excessive substance use in bipolar disorder. Journal of Nervous and Mental Disease, 198, 628-633. doi: 10.1097/NMD.0b013e3181 ef3ef4

Lecrubier, Y., Sheehan, D., Weiller, E., Amorim, P., Bonora, L.I. y Sheehan, K. (1997). The MINI International Neuropsychiatric Interview (MINI) A short diagnostic structured interview: Reliability and validity according to the CIDI. European Psychiatry, 12, 224-231.

Levin, F.R. y Hennessy, G. (2004). Bipolar disorder and substance abuse. Biological Psychiatry, 56, 738-748.

Mantere, O., Melartin, T.K., Suominen, K., Rytsälä, H.J., Valtonen, H.M., Arvilommi, P., Leppämäki, S. y Isometsä, E.T. (2006). Differences in Axis I and II comorbidity between bipolar I and II disorders and major depressive disorder. Journal of Clinical Psychiatry, 67, 584-593.

Maremmani, I., Maremmani, A.G., Rugani, F., Rovai, L., Pacini, M., Bacciardi, S., ... Akiskal, H.S. (2012). Clinical presentations of substance abuse in bipolar heroin addicts at time of treatment entry. Annals of General Psychiatry, 11, 23. doi: 10.1186/1744-859X-11-23

Mazza, M., Mandelli, L., Di Nicola, M., Harnic, D., Catalano, V., Tedeschi, D., ... Janiri, L. (2009). Clinical features, response to treatment and functional outcome of bipolar disorder patients with and without co-occurring substance use disorder: 1-year follow-up. Journal of Affective Disorders, 115, 27-35. doi: 10.1016/j. jad.2008.08.019

McElroy, S.L., Altshuler, L.L., Suppes, T., Keck, P.E. Jr, Frye, M.A., Denicoff, K.D., ... Post, R.M. (2001). Axis I psychiatric comorbidity and its relationship to historical illness variables in 288 patients with bipolar disorder. American Journal of Psychiatry, 158, 420-426. 
Merikangas, K.R., Akiskal, H.S., Angst, J., Greenberg, P.E., Hirschfeld, R.M., Petukhova, M. y Kessler, R.C. (2007). Lifetime and 12-month prevalence of bipolar spectrum disorder in the National Comorbidity Survey replication. Archives of General Psychiatry, 64, 543-552.

Merikangas, K.R., Herrell, R., Swendsen, J., Rössler, W., Ajdacic-Gross, V. y Angst, J. (2008). Specificity of bipolar spectrum conditions in the comorbidity of mood and substance use disorders: results from the Zurich cohort study. Archives of General Psychiatry, 65, 47-52. doi: 10.1001/archgenpsychiatry.2007.18

Merikangas, K.R., Jin, R., He, J.P., Kessler, R.C., Lee, S., Sampson, N.A., ... Zarkov, Z. (2011). Prevalence and correlates of bipolar spectrum disorder in the world mental health survey initiative. Archives of General Psychiatry, 68, 241-251. doi: 10.1001/archgenpsychiatry.2011.12

Mitchell, J.D., Brown, E.S. y Rush, A.J. (2007). Comorbid disorders in patients with bipolar disorder and concomitant substance dependence. Journal of Affective Disorders, 102, 281-287.

Nallet, A., Weber, B., Favre, S., Gex-Fabry, M., Voide, R., Ferrero, F., ... Aubry, J.M. (2013). Screening for bipolar disorder among outpatients with substance use disorders. European Psychiatry, 28, 147-153.

Pacini, M., Maremmani, I., Vitali, M., Romeo, M., Santini, P., Vermeil, V. y Ceccanti, M. (2010). Cocaine Abuse in 448 Alcoholics: Evidence for a Bipolar Connection. Addictive Disorders Eo their Treatment, 9, 164-171.

Powers, R.L., Russo, M., Mahon, K., Brand, J., Braga, R.J., Malhotra, A.K. y Burdick, K.E. (2013). Impulsivity in bipolar disorder: relationships with neurocognitive dysfunction and substance use history. Bipolar Disorders, 15, 876-884. doi: 10.1111/bdi.12124

Regier, D.A., Farmer, M.E., Rae, D.S., Locke, B.Z., Keith, S.J., Judd, L.L. y Goodwin, F.K. (1990). Comorbidity of mental disorders with alcohol and other drug abuse: Result from the Epidemiologic Catchment Area (ECA) Study. The Journal of the American Medical Association, 264, 2511-2518.

Salloum, I.M., Cornelius, J.R., Douaihy, A., Kirisci, L., Daley, D.C. y Kelly, T.M. (2005). Patient characteristics and treatment implications of marijuana abuse among bipolar alcoholics: results from a double blind, placebo-controlled study. Addictive Behaviors, 30, 1702-1708.

Sheehan, D.V., Lecrubier, Y., Harnett-Sheehan, K., Janavs, J., Weiller, E. y Bonora, L.I. (1997). Reliability and validity of the MINI International Neuropsychiatric Interview (MINI): According to the SCID-P. European Psychiatry, 12, 232-241.

Sheehan, D.V., Lecrubier, Y., Sheehan, K.H., Amorim, P., Janavs, J., Weiller, E., Hergueta, T., ... Dunbar, G.C. (1998). The Mini-International Neuropsychiatric Interview (M.I.N.I.): the development and validation of a structured diagnostic psychiatric interview for DSM-IV and ICD-10. Journal of Clinical Psychiatry, 59, 22-33.

Simon, N.M., Otto, M.W., Weiss, R.D., Bauer, M.S., Miyahara, S., Wisniewski, S.R., ... Pollack, M.H. (2004). Pharmacotherapy for bipolar disorder and comorbid conditions: baseline data from STEP-BD. Journal of Clinical Psychopharmacology, 24, 512-520.

Singh, M.K., Chang, K.D., Kelley, R.G., Cui, X., Sherdell, L., Howe, M.E., ... Reiss, A.L. (2013). Reward processing in adolescents with bipolar I disorder. Journal of the American Academy Child E्F Adolescent Psychiatry, 52, 68-83. doi: 10.1016/j.jaac.2012.10.004

Sonne, S.C. y Brady, K.T. (1999). Substance abuse and bipolar comorbidity. Psychiatry Clinics of North America, 22, 609-627.

Stewart, C. y El-Mallakh, R.S. (2007). Is bipolar disorder overdiagnosed among patients with substance abuse? Bipolar Disorders, 9, 646-648.

Tondo, L., Baldessarini, R.J., Hennen, J., Minnai, G.P., Salis, P., Scamonatti, L., ... Mannu, P. (1999). Suicide attempts in major affective disorder patients with comorbid substance use disorders. Journal of Clinical Psychiatry, 2, 63-69.

Trost, S., Diekhof, E.K., Zvonik, K., Lewandowski, M., Usher, J., Keil, M., ... Gruber, O. (2014). Disturbed anterior prefrontal control of the mesolimbic reward system and increased impulsivity in bipolar disorder. Neuropsychopharmacology, 39, 1914-1923. doi: 10.1038/npp.2014.39

Van Zaane, J., Van den Berg, B., Draisma, S., Nolen, W.A. y Van den Brink, W. (2012). Screening for bipolar disorders in patients with alcohol or substance use disorders: performance of the mood disorder questionnaire. Drug and Alcohol Dependence, 124, 235-241. doi: 10.1016/j.drugalcdep.2012.01.018

Weaver, T., Madden, P., Charles, V., Stimson, G., Renton, A. y Tyrer, P. (2003). Comorbidity of substance misuse and mental illness in community mental health and substance misuse services. British Journal of Psychiatry, 183, 304-313.

Yatham, L.N., Kauer-Sant'Anna, M., Bond, D.J., Lam, R.W. y Torres, I. (2009). Course and outcome after the first manic episode in patients with bipolar disorder: Prospective 12-month data from the systematic treatment optimization program for early mania project. Canadian Journal of Psychiatry, 54, 105-112. 